Ann. Biol. anim. Bioch. Biophys., I963, 3 (4), 38I-389

\title{
COMPORTEMENT ÉLECTROPHORÉTIQUE DES PROTÉINES SARCOPLASMIQUES DU MUSCLE DE PORC NORMAL ET EXSUDATIF
}

\author{
J. CHARPENTIER et R. GOUTEFONGEA \\ avec la collaboration technique de Jacqueline PARIS \\ Laboratoire de Recherches sur la Viande, \\ Centre national de Recherches zootechniques, Jouy-en-Josas (Seine-et-Oise)
}

\section{SOMMAIRE}

Cette étude qui a porté sur 30 porcs de race Large W'hite pesant $100 \mathrm{~kg}( \pm 3 \mathrm{~kg})$ a consisté essentiellement en l'examen du comportement électrophorétique des protéines musculaires en milieu de faible force ionique. Conjointement, un certain nombre d'échantillons ont été soumis à un enregistrement de pII pendant 24 heures.

En outre, sur tous les échantillons, le $\mathrm{pH}$, le pouvoir de rétention d'eau et la teneur en pigment total ont été déterminés 24 heures après l'abattage.

L'examen des courbes d'enregistrement de pH permet de classer les animaux en 3 catégories en fonction de la vitesse de chute $\mathrm{du} \mathrm{pH}$. Cette classification correspond à celle établie 24 heures après l'abattage en fonction de l'état exsudatif.

Les électrophorégrammes obtenus ne montrent aucune différence qualitative, ni entre échantillons provenant d'animaux normaux et exsudatifs, ni entre échantillons prélevés aussitôt après l'abattage et 24 heures après, et ceci avec les deux modes de coloration utilisés. D'autre part, nous avons pu identifier un certain nombre des bandes obtenues par les deux modes de coloration.

I'affection connue sous le nom de "Myopathie exsudative et dépigmentaire" du Porc (COSSARD, I957) désigne un ensemble de perturbations biochimiques et physicochimiques du muscle propres à cette espèce. Dans l'état actuel de nos connaissances, ce syndrome ne se traduit par aucune manifestation clinique apparente, mais essentiellement par la modification de quelques caractéristiques physicochimiques du muscle, en particulier une chute du $\mathrm{pH}$ post-mortem rapide (BRISkEY et WISMER-PEDERSEN, I96I), une faible valeur du $\mathrm{pH} 24$ heures après la mort, une teneur en pigment moindre et un pouvoir de rétention d'eat1 plus faible que dans les muscles d'individus normaux. Les myopathies métaboliques que l'on rencontre 
chez d'autres espèces sont caractérisées, d'une part, par diverses manifestations cliniques et, d'autre part, par des modifications de certains constituants protéiques du tissu musculaire (HuGUEs, I960). Le comportement électrophorétique des protéines musculaires et de certaines enzymes est, en particulier, notablement différent chez le sujet "normal " et chez le myopathe (DREYrus et al., I962 ; HAAN, I953; KEELER et YOUNG, I961; SChapira et DREYFus, I958).

Nous avons voulu rechercher si ces modifications se retrouvent chez le porc. exsudatif.

Dans ce but, nous avons comparé les caractéristiques électrophorétiques d'extraits musculaires provenant de sujets normaux et de porcs présentant les déviations physico-chimiques caractéristiques de la myopathie exsudative et dépigmentaire. Nous nous sommes limités dans un premier temps à étudier le comportement des protéines extractibles en milieu de faible force ionique, c'est-à-dire des seules protéines sarcoplasmiques.

Afin d'éviter de choisir les porcs " normaux " et " exsudatifs" en fonction d'un jugement strictement subjectif, nous avons préféré étayer cette qualification par la détermination de certains critères physicochimiques qui sont habituellement utilisés en vue de la caractérisation objective de cette affection.

\section{MATÉRIEL, ET MÉTHODE}

L'étude a porté sur $3^{\circ}$ porcs de race Large White pesant $100 \mathrm{~kg}( \pm 3 \mathrm{~kg})$.

\section{$\mathrm{I}^{\mathrm{O})}$ Techniques}

L'abattage comprenait successivement : électrocution, saignée, échaudage.

Un échantillon de $50 \mathrm{~g}$ des muscles Transiersus abdominis et Gracilis était prélevé I 5 minutes après la fin des opérations. Ces muscles ont été choisis en raison, d'une part, de leur facilité de prélèvement et, d'autre part, de leur différence de pigmentation. Les échantillons étaient broyés dans un omni mixer Servall en présence de 2 volumes de Tampon Tris (Hydroxyméthylaminométhane) à pH 7,65. Après $20 \mathrm{mn}$ de centrifugation à $6000 \mathrm{t} / \mathrm{mn}$ et à environ $15^{\circ} \mathrm{C}$, les surnageants étaient prélevés en vue des insertions.

La méthode utilisée était celle de l'électrophorèse en gel d'amidon selon la technique discontinue de Poulik ( (1957) à pH 8,4 pendant 5 h 30 dans un champ électrique de $6 \mathrm{~V} / \mathrm{cm}$. Les constituants protéiques de l'extrait analysé étaient colorés par l'amidoschwarz. La myoglobine était mise en évidence par sa réaction peroxydasique donnant une coloration brune avec la benzidine. Les électrophorégrammes étaient photographiés dès leur sortie des bains de coloration.

Les carcasses étaient laissées en ressuyage à la température ambiante, soit environ I $5^{\circ} \mathrm{C}$ pendant I heure, puis transportées dans un local où la température était maintenue à $15^{\circ} \mathrm{C}$ et l'hygrométrie le plus près possible de $100 \%$. Elles y étaient conservées pendant 24 heures.

Sur ig de ces carcasses, le pH du muscle Gracilis fut enregistré en continu pendant 24 heures à l'aide d'un pHmètre E. I. L. $23 \mathrm{AF}$ et d'un potentiomètre enregistreur P. E. P.

24 heures après l'abattage, on procédait au classement subjectif des carcasses et au prélèvement d'échantillons (5०g) sur les mêmes muscles que la veille, en vue, d'une part, des déterminations de $\mathrm{pII}$, teneur en pigment et pouvoir de rétention d'eau et, d'autre part, de la préparation d'extraits destinés à l'électrophorèse. Ceci, dans le but d'étudier l'influence éventuelle du développement de la rigor mortis sur le comportement électrophorétique des protéines musculaires.

Les carcasses étaient classées d'après le jugement visuel en 3 catégories :

I : Viande ferme, sèche, rose foncé.

II : Viande de tenue moyenne, rose clair.

III : Viande flasque, exsudat abondant, décoloration très prononcée. 
Le pigment musculaire total (myoglobine et hémoglobine résiduelle) était déterminé par la méthode à l'hématine acide d'HorNSEY (1956).

La mesure du pouvoir de rétention d'eau était effectuée par pression à l'aide de l'appareil de Goutefongea (r960). A l'issue de cette mesure, en traduisait le pouvoir de rétention d'eau par le pourcentage d'eau défini par:

$$
\% \text { eau libre }=\frac{\text { Poids initial de l'échantillon }- \text { poids final }}{\text { Poids initial }} \times 100 .
$$

\section{$\left.2^{\circ}\right)$ Caractérisation des carcasses}

Le tableau I indique les valeurs du $\mathrm{pH}_{24}$ heures, de la teneur en pigment total, du pourcentage d'eau libre des deux muscles ainsi que la catégorie dans laquelle les carcasses sont classées d'après le jugement subjectif.

\section{TABLEAU I}

Résultats du jugement subjectif et des différentes mesures effectuées sur les 30 carcasses

\begin{tabular}{|c|c|c|c|c|c|c|c|}
\hline \multirow{2}{*}{ Porc $n^{0}$} & \multirow{2}{*}{ Catégorie } & \multicolumn{2}{|c|}{ pII 2-k h } & \multicolumn{2}{|c|}{$\begin{array}{c}\text { Pigment total en } \mathrm{mg} / \mathrm{g} \\
\text { de tissu frais }\end{array}$} & \multicolumn{2}{|c|}{$\begin{array}{c}\text { Taul libre en } \% \\
\text { de tissu frais }\end{array}$} \\
\hline & & A & $B$ & A & $\mathrm{B}$ & A & $B$ \\
\hline $1 \ldots \ldots \ldots$ & III & 5,29 & 5,55 & 0,62 & 2,47 & 35,29 & 17,08 \\
\hline $2 \ldots \ldots \ldots \ldots$ & III & 5,35 & 5,55 & 0,50 & 2,50 & 24,23 & 21,82 \\
\hline $3 \ldots \ldots \ldots \ldots$ & III & 5,35 & 5,59 & 1,00 & 3,91 & 27,74 & 22,94 \\
\hline $4 \ldots \ldots \ldots \ldots$ & III & 5,36 & 5,71 & 0,47 & 2,23 & 27,06 & 21,45 \\
\hline $5 \ldots \ldots \ldots \ldots$ & II & 5,40 & - & 1,03 & 2,31 & 20,92 & 27,51 \\
\hline $6 \ldots \ldots \ldots$ & II & 5,40 & 5,62 & 0,75 & 3,78 & 24,21 & 28,08 \\
\hline $7 \ldots \ldots \ldots$ & III & 5,40 & 5,71 & 0,68 & 3,23 & 19,07 & 27,80 \\
\hline $8 \ldots \ldots \ldots$ & II & 5,41 & 5,71 & 1,77 & 3,23 & 21,06 & 24,50 \\
\hline $9 \ldots \ldots \ldots$ & II & 5,45 & - & - & - & 22,40 & 15,31 \\
\hline $10 \ldots \ldots \ldots \ldots$ & III & 5,47 & 5,60 & 1,16 & 2,75 & 29,03 & 18,97 \\
\hline $11 \ldots \ldots \ldots$ & II & 5,48 & 5,62 & 0,86 & 2,89 & 18,58 & 16,72 \\
\hline $12 \ldots \ldots \ldots \ldots$ & II & 5,50 & - & - & - & $20,6^{\prime}$ & 20,59 \\
\hline $13 \ldots \ldots \ldots \ldots$ & III & 5,50 & 5,65 & 0,70 & 2,30 & 34,05 & 14,62 \\
\hline $1 ' \ldots \ldots \ldots \ldots$ & I & 5,50 & 5,68 & 1,00 & 3,24 & 17,98 & 18,72 \\
\hline $15 \ldots \ldots \ldots \ldots$ & III & 5,50 & 5,70 & 0,85 & 2,24 & 32,58 & 22,64 \\
\hline $16 \ldots \ldots \ldots \ldots$ & II & 5,50 & 1,75 & 1,00 & 2,90 & 20,92 & 19,22 \\
\hline $17 \ldots \ldots \ldots$ & I & 5,50 & 5,90 & 1,23 & 3,37 & 13,93 & 10,07 \\
\hline $18 \ldots \ldots \ldots \ldots$ & II & 5,52 & 5,70 & 1,17 & 2,13 & 17,76 & 18,46 \\
\hline $19 \ldots \ldots \ldots \ldots$ & II & 5,53 & 5,52 & 1,25 & 2,47 & 25,45 & 22,00 \\
\hline $20 \ldots \ldots \ldots \ldots$ & II & 5,55 & 5,75 & 1,02 & 2,42 & 19,59 & 14,44 \\
\hline $21 \ldots \ldots \ldots \ldots$ & III & 5,55 & - & 0,65 & 2,70 & 31,10 & 22,57 \\
\hline $22 \ldots \ldots \ldots \ldots$ & II & 5,56 & 5,72 & 1,32 & 3,63 & 24,39 & 17,64 \\
\hline $23 \ldots \ldots \ldots$ & III & 5,58 & 5,67 & 0,32 & 2,07 & 26,69 & $2 !, 51$ \\
\hline $2+\ldots \ldots$ & II & 5,58 & 5,70 & 0,74 & 2,18 & 24,24 & 13,52 \\
\hline $25 \ldots \ldots$ & II & 5,60 & 5,82 & 0,81 & 2,44 & 19,09 & 12,36 \\
\hline $26 \ldots \ldots$ & $\mathrm{I}$ & 5,60 & 6,00 & 1,40 & 3,66 & 20,95 & 13,66 \\
\hline $27 \ldots \ldots \ldots \ldots$ & $\mathrm{I}$ & 5,70 & 5,75 & 1,61 & 3,46 & 21,80 & $14,1^{\prime}$ \\
\hline $28 \ldots \ldots \ldots \ldots$ & I & 5,90 & 6,35 & 0,93 & 2,43 & 17,03 & 6,94 \\
\hline $29 \ldots \ldots$ & I & 5,91 & 5,95 & 1,00 & 2,98 & 11,64 & 9,66 \\
\hline $30 \ldots \ldots \ldots \ldots$ & I & 6,00 & 6,50 & 0,97 & 2,57 & 12,11 & 9,84 \\
\hline
\end{tabular}

$\mathrm{A}=$ muscle Gracilis.

$\mathrm{B}=$ muscle Transwersus abdominis. 
La vitesse de chute du pHI post-morlem du muscle Gracilis nous permet de distinguer 3 types d'évolution (fig. I) ;

$\left.1^{\circ}\right) \mathrm{La}$ chute du $\mathrm{pH}$ est lente. La pH atteint une valeur stable (comprise entre 5,50 et 6,00) après 12 heures environ.

$\left.2^{\circ}\right)$ La chute du pH est rapide pendant les 3 premières heures. Le pH se stabilise ensuite à une valeur comprise entre 5,50 et 5,70 .

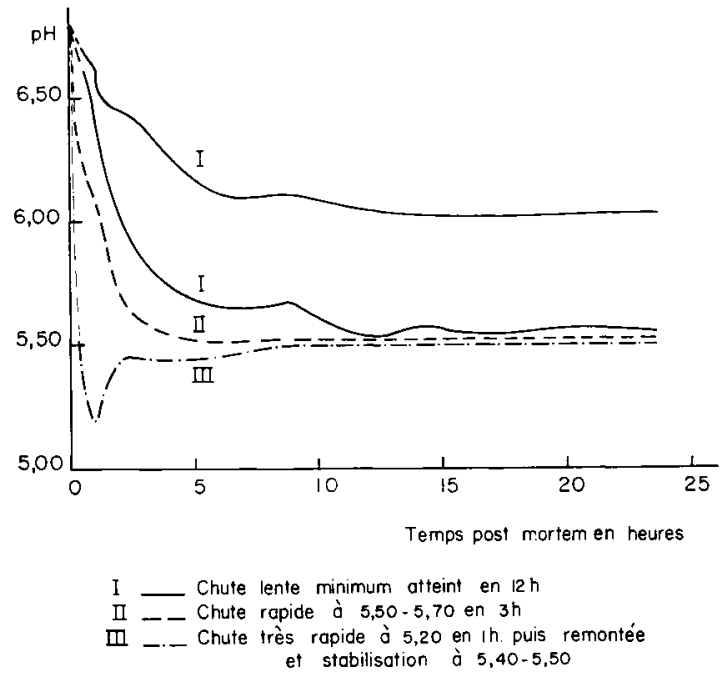

FIG. I. - Différents types d'évolution post mortem du $p H$ dans le musscle gracilis

$\left.3^{\circ}\right)$ La chute du pH est très rapide. Au bout d'une heure, le pII est inférieur à 5,30 , puis il remonte plus ou moins rapidement pour se stabiliser aux environs de 5,40 à 5,50.

Les animaux dont le $\mathrm{pH}$ du muscle Gracilis a été enregistré pendant les 24 heures suivant l'abattage se répartissent dans les 3 types définis ci-dessus comme l'indique le tableau 2.

\section{TABI,LAU 2}

Classification des porcs ayant subi l'enregistrement de pII dans les 3 catégories définies par la figure I

\begin{tabular}{|c|c|}
\hline Catérgorie & Pores numéros \\
\hline $1 \ldots \ldots \ldots \ldots \ldots$ & $14-17-20,-27-29-30$ \\
\hline$\because \ldots \ldots$ & $5-6,9-12-18-20-2 x^{\prime}$ \\
\hline$s^{\prime} \ldots \ldots \ldots \ldots \ldots$ & $1-2-3-13-15-21$ \\
\hline
\end{tabular}

In tenant compte de l'ensemble des différents critères étudiés (jugement subjectif, vitesse de chute du $\mathrm{pH}, \mathrm{pH}$ ultime, teneur en pigments et pourcentage d'eau libre) nous pouvons penser que les porcs $17,27,30$ peuvent être considérés comme "normaux » et les porcs I, 2, I3, I5, 2I, comme nettement " exsudatifs". 


\section{RÉSULTATS - DISCUSSION}

Les électrophorégrammes obtenus à partir d'échantillons musculaires prélevés aussitôt après l'abattage ou 24 heures après, sont identiques.

Comme le montre la figure 2 , les électrophorégrammes d'extraits de muscles " normaux " et " exsudatifs" ne présentent pas de différences apparentes.
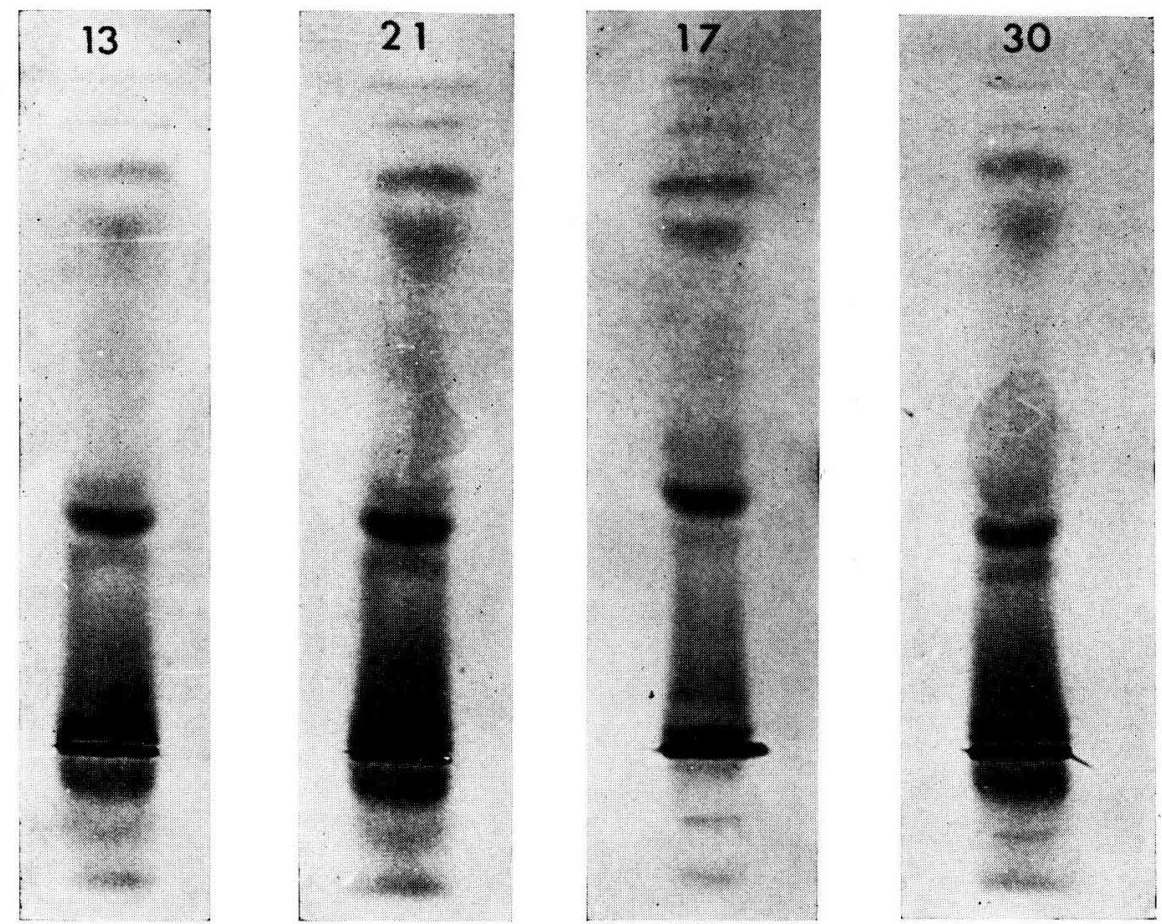

FIG. 2. - Les électrophorégrammes de porcs classés normaux (1 7-30) el de porcs classés exsulatifs ( 1 3-2 I) ne présentent pas de difiérences

Dans tous les cas par la coloration à l'amidoschwarz, il est possible de mettre en évidence (plus ou moins nettement) I5 bandes dont 3 migrent vers la cathode et I2 vers l'anode (fig. 3 ).

Les bandes correspondantes des différents muscles ont une vitesse de migration semblable.

La coloration peroxydasique à la benzidine montre 6 bandes (fig. 3) dont une cathodique et cinq anodiques. Les deux bandes anodiques les plus rapides sont très faibles et parfois difficiles à mettre en évidence. Les deux bandes moyennes sont plus larges et plus intenses. Quant à la bande anodique la plus lente, elle est très faible et souvent à peine visible.

Les bandes colorées à la benzidine présentent dans tous les cas des vitesses de migration semblables, mais leur nombre n'est pas constant. 
En effet, les bandes A, C, D sont toujours visibles, mais la bande B, d'une part, et les bandes $\mathrm{F}$ et $\mathrm{F}$, d'autre part, ne sont souvent que peu perceptibles. Ces bandes sont d'autant plus difficiles à déceler que la teneur en myoglobine des muscles est plus faible. La concentration d'extraits aqueux par lyophilisation nous a toutefois permis de mettre en évidence l'existence de ces bandes dans les muscles faiblement pigmentés.
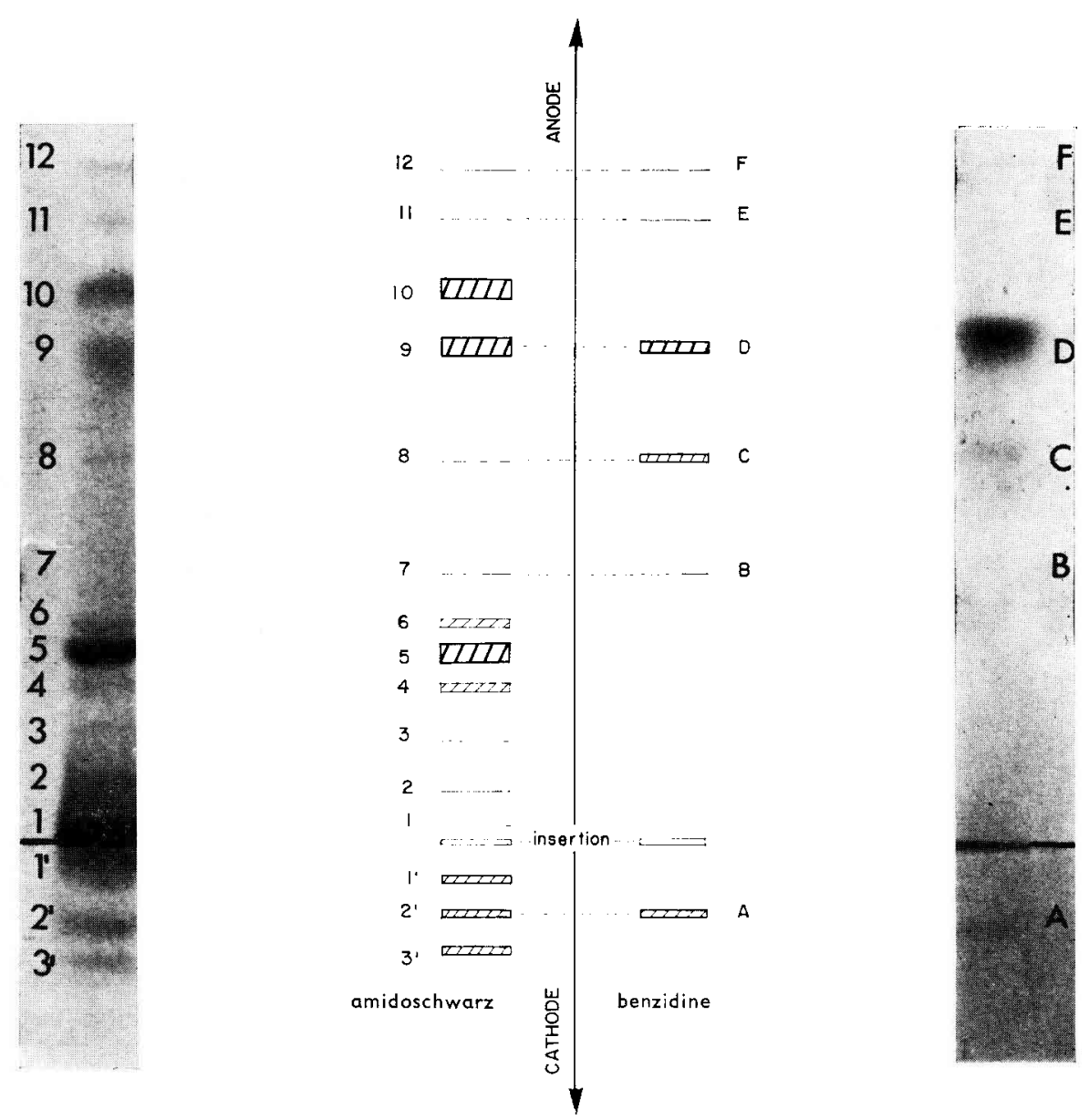

FIG. 3. - Disposition des différentes bandes mises en évidence par les deux modes de colorationt et correspondance de ces bandes entre elles

La confrontation des électrophorégrammes obtenus par coloration à l'amidoschwarz, d'une part, et à la benzidine, d'autre part, semble montrer l'analogie des bandes $2^{\prime}$ et $\mathrm{A}, 7$ et $\mathrm{B}, 8$ et $\mathrm{C}, 9$ et $\mathrm{D}$, II et $\mathrm{E}$, I2 et $\mathrm{F}$ (fig. 3).

La bande 9 -D correspond à la chromoprotéine la plus importante dans le tissu musculaire, c'est-à-dire à la myoglobine. Il est probable que les bandes I I-E et I2-F correspondent également à la myoglobine. En effet, BERNARD et al. (I96I) ont mis en évidence pour la myoglobine de porc l'existence d'une bande anodique intense 

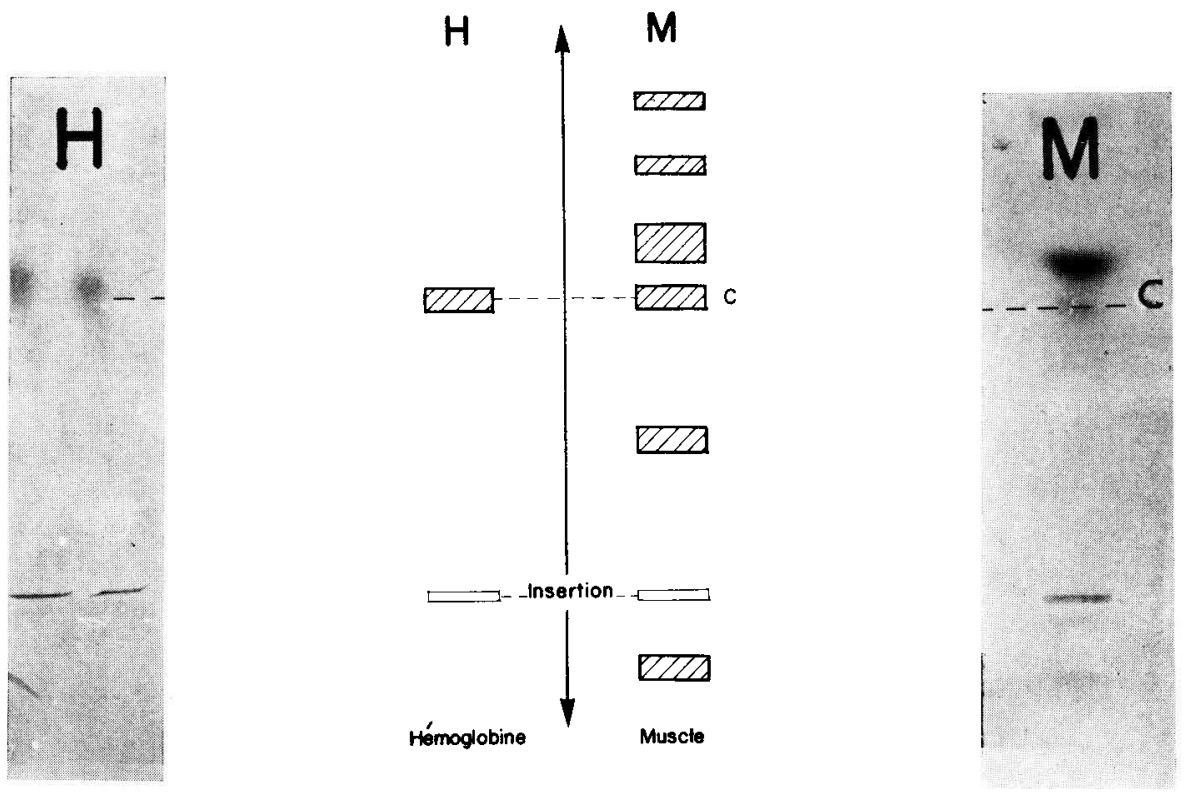

Fig. 4. - La bande C révélée à la benzidine correspond a l'hémoglobine du muscle $\mathrm{H}=$ hémoglobine $\mathrm{M}=$ muscle
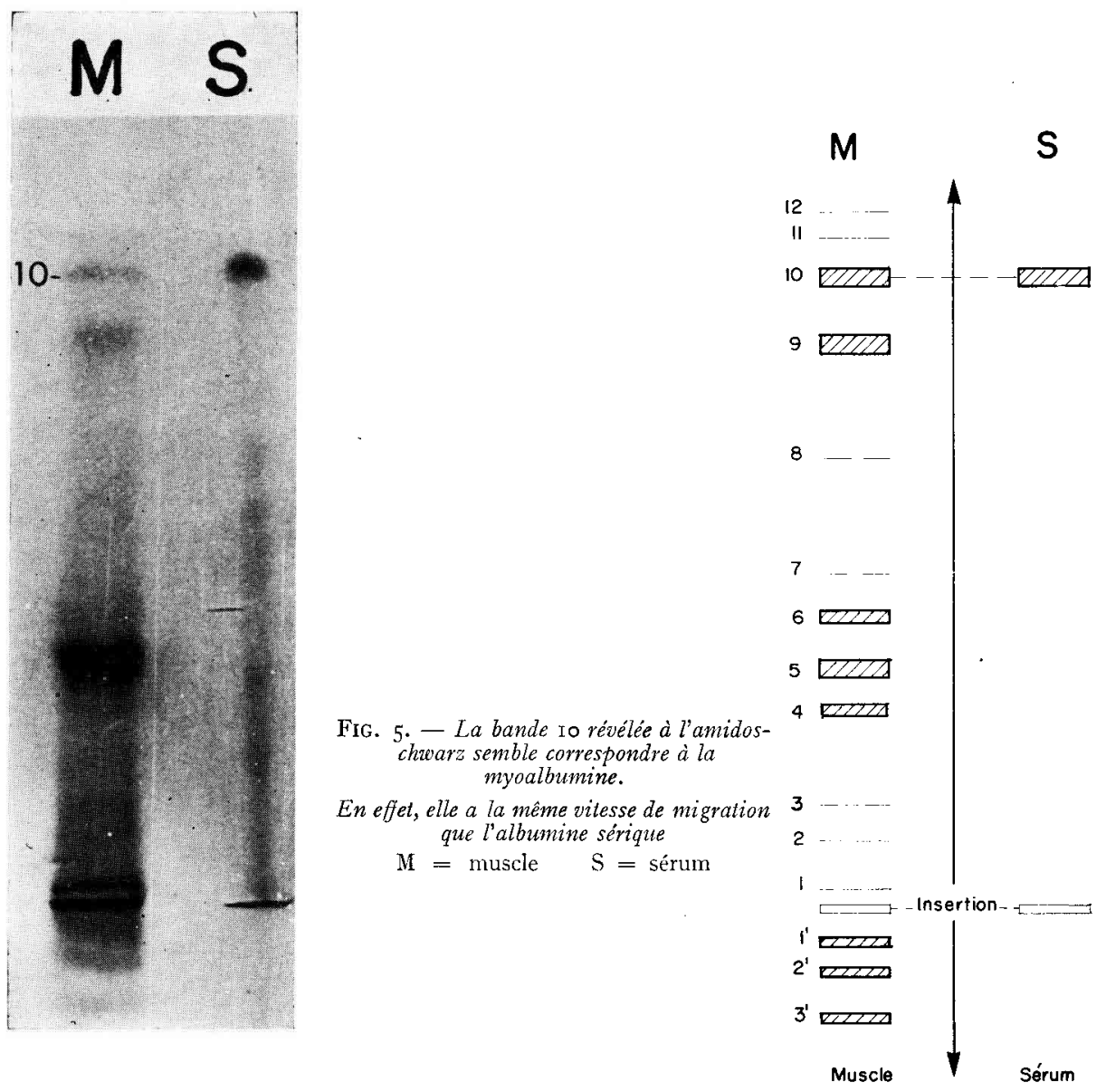

FIg. 5. - La bande ro révélée à l'amidoschwarz semble correspondre à la myoalbumine.

En effet, elle a la même vilesse de migration que l'albumine sérique $\mathrm{M}=$ muscle $\mathrm{S}=$ sérum 
et de deux bandes de très faible intensité et de vitesse de migration supérieure.

L'électrophorèse comparative d'extrait musculaire et d'hémoglobine nous a montré (fig. 4) que la bande 8 -C représentait l'hémoglobine résiduelle đu tissu musculaire.

La nature de la bande 7-B ne peut être précisée. La bande 2'-A semble, par contre, correspondre au cytochrome C. L'électrophorèse d'une solution pure de cytochrome $\mathrm{C}$ nous a montré, en effet, que cette chromoprotéine présentait une migration cathodique de même vitesse que la bande $2^{\prime}-\mathrm{A}$.

La bande Io semble correspondre à la myoalbumine. Sa vitesse de migration est identique à celle de 1'albumine plasmatique (fig. 5).

L'état actuel de nos connaissances ne permet pas d'identifier les autres bandes.

\title{
CONCLUSION
}

Chez le porc présentant post mortem les signes de l'affection connue sous l'appellation de "myopathie exsudative et dépigmentaire », l'électrophorèse en gel d'amidon des protéines musculaires extractibles en milieu de faible force ionique, ne fournit pas, dans nos conditions expérimentales, un électrophorégramme différent de celui du porc "normal». Le nombre et la position des bandes ne sont pas modifiés. La difficulté de révélation de certaines bandes de faible intensité semble essentiellement imputable à des variations de concentration des protéines dans les extraits. Il se pourrait donc que la répartition quantitative de ces diverses fractions protéiques extractibles soit modifiée.

I1 semble néanmoins que l'affection considérée ne présente pas d'analogies avec certaines myopathies, telles que les dystrophies musculaires pour lesquelles divers constituants protéiques du muscle présentent un comportement électrophorétique caractéristique et différent de la normale.

Toutefois, il est possible que, dans cette myopathie, seules les protéines de faible solubilité puissent présenter des anomalies, aussi paraît-il indispensable de compléter ultérieurement l'étude électrophorétique des protéines sarcoplasmiques par celle des protéines de structure telles que l'actine et la myosine.

\section{Reçu pour publication en juillet 1963 .}

\author{
SUMMARY \\ ELECTROPHORETIC BEHAVIOUR OF SARCOPLASMIC PROTEINS OF NORMAL \\ AND EXUDATIVE MUSCLE IN THE PIG
}

The aim of this study was to see whether « Myopathie exsudative et depigmentaire " (Muscular Degeneration) in the pig can be distinguished by modifications in the electrophoretic behaviour of certain sarcoplasmic proteins such as those which occur in some myopathies met with in other species. This work was carried out with $3^{\circ}$ pigs of the Large White breed, weighing $100 \mathrm{~kg}( \pm 3 \mathrm{~kg})$.

The work was essentially based on an examination of the electrophoretic behaviour (starch-gel electrophoresis, with a discontinuous buffer system, under a 6 volts $/ \mathrm{cm}$ voltage for $5 \mathrm{~h} 30 \mathrm{mins}$ ) of the proteins extracted a low ionic strength, from samples of the Gracilis and Transversus Abdo. 
minis muscles. Two tests were made on each muscle : the first inmediately after slaughtering, the second 24 hours later. The electrophoretic strips were coloured by two methods : Amidoschwarz and Benzidine. In addition, most of the samples of Gracilis muscle were submitted to a $\mathrm{pH}$ recording during the 24 hours following slaugtering. Moreover, in all the samples, the $\mathrm{pH}$, water-holding capacity, and total amount of pigment were determined 24 hours after slaughtering (table $\mathrm{I}$ ).

From the pli curves, the animals can be classed under three categories in terms of the speed of fall of the $\mathrm{pH}$. This classification corresponds to that established for 24 hours after slaugther in terms of the exudative state (fig. I, table 2 ).

The electrophoretic strips obtained show no qualitative difference, either among the samples from normal and exudative animals (fig. 2), or among samples taken immediately after slaughtering and 24 hours later, even with two methods of coloration being used.

We have shown 5 bands by coloration with Amidoschwarz and 6 derived from the Benzidine method, these last corresponding moreover to the bands obtained Amidoschwarz (fig. 3). Furthermore, we have been able to identify a certain number of the bands obtained by the two methods of coloration (fig. 4 and 5 ).

\section{RÉFÉRENCES BIBLIOGRAPHIQUES}

Bernard S., Havez R., Daftrevaux M., Biserte G., igor. Étude sur la myoglobine. III. Propriétés comparées des myoglobines de Cheval, de Bouf, de Mouton et de Porc. Bull. Soc. Chim. Biol., 43, I 281 I-I 287.

Briskey E. J., Wismer-Pedersen J., I96I. Biochenistry of pork muscle structure. I. Rate of anaérobic glycolysis and temperature change versus the apparent structure of muscle tissue .J. Food. Sci., 26, $297-305$.

Cossard J., I957. La myopathic exsudative et dẹpirmentaire du Porc, 62 p. Thèse Inctorat Vélérinaire, Paris.

Dreyrus J. C., Demos J., Scirapira F., Schapira G., I962. La lacticodéshydrogénase chez le myopathe: persistance apparente du type foetal. C.R. Acad. Sci., 254, 4384-4386.

Goutefongea R., 1960. Description d'un nouvel appareil pour mesurer le pouvoir de rétention d'eau de la viande. Sixth Meeting Meat Res. Instit. Utrechl.

HAAN A. M. F. II., 1953. Electrophoresis of the non structural proteins from normal and atrophic muscles of the rabbit and of man. Biochim. Biophys. Acta, 11, 258-269.

HoRnsey H. C., 1956. The colour of cooked cured Pork. I. Estimation of the nitric oxide-haem pigments. J. Sci. Food. Agric., 9, 534-540.

Hugues B. P., i 960 . Effects of degeneration on the albumin components of human muscle extracts. Nature, 188, I020-I029.

Keeler R. F., Young S., I96r. An electrophoretic analysis of protein extracts from normal and dystrophic ovine muscle. Biochem. J., 81, 93-98.

Poulik M. D., I957. Starch gel electrophoresis in a discontinous system of buffers. Nature, 180, I477-I 479

SChapira G., Dreyfus J. C., 1958. Electrophorise des protéines musculaires solubles en milieu de faible force ionique. Souris normales et myopathes. C. R. Soc. Biol., 152, $1705^{-1} 707$. 